\title{
Random walker with improved weighting function for interactive medical image segmentation
}

\author{
Lim Khai Yin and Mandava Rajeswari* \\ School of Computer Science, Universiti Sains Malaysia, 11800, Penang, Malaysia
}

\begin{abstract}
To segment an image using the random walks algorithm; users are often required to initialize the approximate locations of the objects and background in the image. Due to its segmenting model that is mainly reflected by the relationship among the neighborhood pixels and its boundary conditions, random walks algorithm has made itself sensitive to the inputs of the seeds. Instead of considering the relationship between the neighborhood pixels solely, an attempt has been made to modify the weighting function that accounts for the intensity changes between the neighborhood nodes. Local affiliation within the defined neighborhood region of the two nodes is taken into consideration by incorporating an extra penalty term into the weighting function. Besides that, to better segment images, particularly medical images with texture features, GLCM variance is incorporated into the weighting function through kernel density estimation (KDE). The probability density of each pixel belonging to the initialized seeds is estimated and integrated into the weighting function. To test the performance of the proposed weighting model, several medical images that mainly made up of 174-brain tumor images are experimented. These experiments establish that the proposed method produces better segmentation results than the original random walks.
\end{abstract}

Keywords: Random walks, weighting function, gray-level co-occurrence, kernel density estimation

\section{Introduction}

Image segmentation is the process of clustering an image into regions based on some homogeneity criteria. It is usually not hard to extract a region of interest (ROI) when it has distinctively discernible homogeneous features such as color, texture and shape from the background. However, in medical images depending on the pathological tissues or location, the appearance or the substructure of the ROI may not always appear to be prominent. The gradual changes of intensity or inhomogeneity of pixels within a local appearance may hinder an effective segmentation. The ROI may be homogenous in its center, but this homogeneity tends to fade and blend into its background when it is reaching the boundary. This non-uniformity poses major challenge to the satisfactory segmentation of the ROI. In such instances, segmentation methods that possess the capability of handling weak boundaries are often favored.

\footnotetext{
${ }^{*}$ Corresponding author: Mandava Rajeswari, School of Computer Science, Universiti Sains Malaysia, 11800, Penang, Malaysia. Tel.: +604 6532157/4393; Fax: 604-6573335; E-mail: mandava@cs.usm.my.
}

0959-2989/14/\$27.50 @ 2014 - IOS Press and the authors. 
Random walks algorithm, which is equivalent to solving the problem of Dirichlet [1] is among the most common approaches in segmenting images with vague edges. In random walks algorithm, the solution to the discrete Dirichlet problem on a graph is portrayed using the explanation on circuit theory. Associating a pixel to a labeled pixel or seed pixel representing an ROI is solved through finding the probability of a walker from the unlabeled pixel first reaches a priory labeled pixel.

Over the years, there are numerous publications that apply and extend the random walks algorithm to image segmentation. One most frequent improvement done is on automating the semi-automatic random walks algorithm. Instead of manually identifying and labeling the seed pixels of foreground and background, research on automating the seeding process has been addressed in [2-4]. Besides that, several studies have also been made to incorporate an extra term particularly the prior knowledge into the random walks algorithm so as to automate and enhance the results of random walks algorithm [57]. In these studies, the 'aspatial' energy term obtained from the training images or input from the user was integrated into either the conventional spatial energy function or the distance function in the random walks.

The levels of contrast and the properties of the image, as well as the quantity or extensiveness of the seeds are among the factors that influence the random walks segmentation. Segmentation of ROI with better accuracy may be achieved by increasing the extensiveness or quantity of the seeds. However, this increases the necessity for user interaction that is not desirable. Minimum seed input with good output is comparatively preferred. Therefore, to enhance the segmentation results without entailing a change of quantity or locations of the seeds becomes the main focus of this work.

Despite the enormous amount of studies on extending and enhancing the random walks algorithm that are mostly problem-specific, the general issue, sensitivity to seeds' selection is yet to be addressed. Even though it is anticipated [8] that a small deviation on the seeds placement will not cause a large difference in the results, but to enhance the robustness of random walks in ensuring the credibility of the segmentation is undoubtedly indispensable. In random walks algorithm, image is treated as a weighted graph. The probability of a pixel belonging to the seeds relies on the neighborhood pixel intensities that are mapped into edge weights by using a typical Gaussian weighting function. Thus, to solve Dirichlet problem using a system of linear equations, when user input persists, the effect of edges and the corresponding weights between vertexes that constitute the Laplacian matrix is important to be studied. Several studies [9-11] have attempted various weighting functions in improving the random walks segmentation. For instance, Dakua and Sahambi [10] proposed to use the derivative of Gaussian (DRoG) instead of the Gaussian weighting function [1] to segment the ROI with inhomogeneous and indistinctive gray values in ischemic Cardiac Magnetic Resonance (CMR) images. In their work, the derivative of the Gaussian function obtained from [12] was simplified and utilized as the weighting function on the scaled image. Though the proposed weighting functions [9-11] showed generally better results in the segmentation, the tangible properties of the proposed functions were not being addressed in the context of image segmentation. That is the basis of the projected modifications did not take full advantage of the characteristics of the image itself.

Improving segmentation results without increasing the quantity of seeds is the objective of this work. To this end, a modified weighting function of the random walks algorithm that utilizes additional characteristics of the image is proposed. The modifications are, (i) incorporating a penalty term, which analyzes the local relationship within the defined region of the two distant nodes (refer to Section 2). (ii) incorporating an object-feature based affinity, in specific Haralick textural feature, into the edge weights function. 
The rest of the paper is organized as follows: Section 2 briefly reviews the theoretical concepts of the random walks algorithm. Section 3 introduces the proposed model. Section 4 includes experiments and its results and finally Section 5 concludes this paper.

\section{Random walks}

In the state of local finite, e.g. a graph, random walks theory is a mathematically formalized algorithm of finding the probability of a random walker reaching a predefined node. A graph, $G$ is made up of vertices, $v \in V$ and edges, $e \in E \subseteq V \times V, G=(V, E)$. A weighted graph contains weight, $w_{i j}$, at each and every edge, $e_{i j}$ that links $v_{i}$ and $v_{j}$. The number of edges, $e_{i j}$ incident to vertex, $v_{i}$ is known as the degree, $d_{i}=\sum w\left(e_{i j}\right)$.

In image segmentation, the relationship between random walks and Dirichlet problem is established in clustering the respective sub-regions according to the users' inputs [1]. Given a set of foreground seeds, $V_{F g}$, and background seeds, $V_{B g}$, where $V_{S}=V_{F g} \cup V_{B g}$ and $V_{F g} \cap V_{B g}=\emptyset$, the probability of a random walker, $x_{i}$ starting at node $v_{i}$ first reaches a seeded node, $v_{S}$ is equivalent to the solution to the Dirichlet problem of finding the harmonic function subjects to its boundary values.

$$
L_{U} x_{U}=-B^{T} x_{M}
$$

where $L_{U}$, the unseeded nodes in Laplacian, is one component of the decomposition of the combinatorial Laplacian matrix, Eq. (2), $B$ is the boundary conditions at the locations of the seeded points, $x_{M}$.

$$
L_{i j}\left\{\begin{aligned}
d_{i}, & \text { if } i=j \\
-w_{i j}, & \text { if } i \neq j, n_{i} \text { and } n_{j} \text { are adjacent nodes } \\
0, & \text { otherwise }
\end{aligned}\right.
$$

The derivation of Eq. (1) can be found in [1]. With a defined set of seeds $x_{U}$, the belongingness of an unlabelled node $v_{i}$ to the seed $v_{S}$ with label $s$, where $s=\{F g, B g\}$ can be identified when its probability, $p r$ to reach $v_{S}$ with label $s$ is higher.

$$
v_{i}=s \because p r_{i}=\max (s)
$$

The weighting function is represented by the typical Gaussian weighting function,

$$
w_{i j}=\exp \left(-\beta \sqrt{\left(g_{i}-g_{j}\right)^{2}}\right)
$$

where $g_{i}$ indicates the image intensities at $v_{i}$ and the value $\beta$ represents the free parameter that is to be decided by the user. 


\section{Proposed model}

Eq. (1) typifies that the harmonic function changes according to the boundary conditions as well as the variations of it. Therefore, the correct localization of the seeds (boundary conditions) from the user becomes crucial in order to obtain good segmentation. To enhance the result where the user input appears to be the fixed input in the context of the equation, the effect of the edges that contribute to the combinatorial Laplacian matrix on the solution is one useful entity to study [13].

\subsection{Modified weighting function}

The global relationships Eq. (1) between the nodes are initiated by its local connectivity represented in Eq. (2). With the intention of enhancing the robustness of the algorithm regardless of the extensiveness of the user input; the commonly used direct neighborhood of $g_{i}$ and $g_{j}$ is extended to a wider range within some fixed radius, $R$. It is commonly perceived that the stronger the connectivity (more edges) in the graph, the better the segmentation is [14]. However, when the range is extended to more than the direct neighborhood, the information of the intermediate nodes is not considered. This inadequacy causes some loss of information that may afflict the overall result.

Although it is generally assumed that pixels from the same region often share similar intensities, it is not always true in real images. Despite the large intensity difference between the pixels; it still may happen that both the pixels belong to the same region when the intensities of the bounded pixels stay close to the local mean. The undulated weights resulting from merely the intensity differences between nodes may cause higher inter-region similarity that consequently induces negative effects on the segmentation results. Hence, it is suggested to include the statistical dispersion of the intermittent intensities in ensuring a more credible affiliation between the distance nodes. A penalty term is included to reflect a concern on the intensities of the intermediate nodes.

$$
w_{i j}=\exp \left(-\beta \sqrt{\left(g_{i}-g_{j}\right)^{2}}\right) \times P\left(g_{i j}\right)
$$

$P$ is a function that penalizes the dispersion of Gaussian-filtered intensities,

$$
P\left(g_{i j}\right)=\frac{1}{\sqrt{\frac{1}{\mathrm{~N}-1} \sum_{a=1}^{N}\left(G z_{a}-G \bar{z}\right)^{2}}}
$$

where $N=$ cardinality of $\alpha_{i j}=\left|x_{j}-x_{i}\right| \times\left|y_{j}-y_{i}\right|$, the area of the Euclidean geometry formed by $v_{i}$ and $v_{j}$, and $G z_{a}$ is the value of the Gaussian smoothed map at $a$ with $a \in \alpha_{i j}$, and $G \bar{z}$ is the Gaussian mean value in $\alpha_{i j}$. Gaussian filter kernel is used to reduce the responsiveness of the variation of intensities in this penalty term. By multiplying the Gaussian weighting function with the reciprocal of the standard deviation (the denominator of Eq. (6)), finer result with the additional information that better explains the relationship between nodes is expected. Weights that exhibit stronger connectivity within cluster could be advantageous for the segmentation. This modification can be demonstrated by the higher intra- and lower inter- cluster similarity (the right column of Figure 1) resulted from the higher gap between the high and low values of weights attributable to the multiplicative effect in Eq. (5). 
The intuition of segmentation is to divide an image into the corresponding regions based on the similarities between nodes, and weights between them usually best demonstrate it. Inspired by the concept of spectral partitioning that uses the eigenvector and eigenvalues to bisect graphs, the second smallest eigenvalue, Fiedler value, $\lambda_{2}$ is employed to measure the connectivity of the graph. In general, a graph is connected if $\lambda_{2}>0$ and it is usually perceived that small Fiedler value, $\lambda_{2}$ leads to good ratio cut [15]. Therefore, to further assess the weighting functions, $\lambda_{2}$ extracted from the $L_{U}$ is used to measure the separability of the corresponding graph. The smaller the Fiedler value is, the more distinguishable the clusters are.

As depicted in Figure 1, the enhanced weighting function can generate better probability maps with smaller $\lambda_{2}$ values.

\subsection{Object-feature based affinity}

In addition to introducing a penalty term described in the previous section, an object feature based affinity, a notion borrowed from [16] is integrated into the weighting function of Eq. (5). This incorporation is to further represent the contextual information of the ROI.

Object feature based affinity, as the name implies, considers pixels' similarity based on the information of the seeds. Instead of solely depending on the intensity differences in attaining the affinity between nodes, the estimated probability density of the nodes belonging to the feature distribution of a pre-identified priori (seeds with labels) is integrated into the weighting function. For simplicity, Gaussian kernel is employed to produce the prior model that constitutes the estimated densities of each of the priori [5]. The obtained prior model is incorporated into the weighting function [7] instead of the energy function as originally proposed in [5].

Several works [7] have employed image features such as intensity and color in estimating the probabilities. In medical images, segmentation using gray level alone does not always provide the desired results. These images, on the other hand often possess a large amount of textures information that differentiates the regions $[17,18]$. As a result, this work proposes to use the texture property that better represents medical images in generating the probability density.

Image

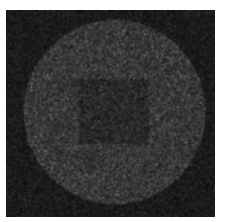

(a)

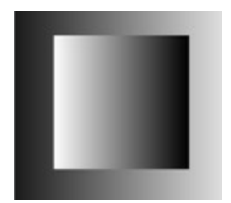

(b)
Original Random walks [1]

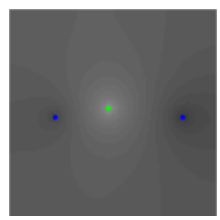

$\lambda_{2}=0.0184$

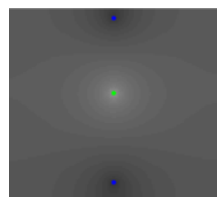

$\lambda_{2}=0.0200$
Random walks with the proposed weighting function

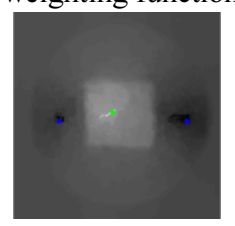

$\lambda_{2}=9.0745 \mathrm{e}-005$

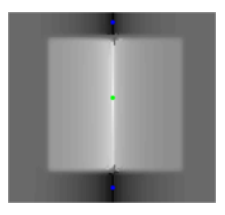

$\lambda_{2}=1.9150 \mathrm{e}-005$

Fig. 1. The probabilities maps of the synthetic images (a) speckle noise (b) gradient image using the conventional and the proposed weighting function respectively. 
In compliance with the literature, it is found that different texture descriptors distinguish different texture discriminants. The Haralick parameters computed from the gray level co-occurrence matrices (GLCM) is chosen to demonstrate the incorporation of textural information into random walks algorithm as it is proven to be one of the most powerful descriptors in feature extraction. Despite having fourteen features in the GLCM, only GLCM variance that measures the heterogeneity is used. The characteristic that captures intensity difference in stochastic texture is suitable when variance is being portrayed as the diversity between regions. GLCM variance is given by,

$$
\text { var }=\sum_{i=0}^{G-1} \sum_{j=0}^{G-1}(i-\mu)^{2} \cdot g(i, j)
$$

where $G$ is the number of gray-level quantization, $g$ is the GLCM, and $\mu$ is the mean value of $g$. Alternately, Eq. (7) may be substituted with other textural features that carry similar characteristic.

To calculate the probability density that the nodes in the image, $I_{i}$ are grouped as the object or background, the values obtained from GLCM variance is used. Given a set of seeds, $S=\left\{s_{1}, s_{2}, \ldots, s_{d}\right\}$ with corresponding labels, $L=\left\{l_{1}, l_{2}, \ldots, l_{c}\right\}$, having cardinality $|S|=d$ and $|L|=c$, the probability, $P_{i}$ of a node belongs to $l_{m}$ is generated using kernel density estimation (KDE).

$$
\hat{f}\left(I_{i}\right)=P_{i}^{l_{m}}=\frac{1}{n} \sum_{i, j=1}^{n} K\left(\frac{I_{i}-s_{j}}{b}\right)
$$

where $b$ is a free parameter while $K$ is the kernel function (typically a Gaussian, Eq. (8)) satisfying $K(x) \geq 0$ and $\int K(x) d x=1$, while $s_{j}$ and $n$ are the values of the seeds' variance and the number of seeds in $l_{m}$ respectively.

$$
K(u)=\exp \left(-\frac{1}{2} u^{2}\right)
$$

When each node has obtained its $P_{i}$, the weights between nodes can be computed by using its differences,

$$
w_{i j}^{p}=\exp \left(-\beta \sqrt{\left(P_{i}-P_{j}\right)^{2}}\right)
$$

The final weighting function will be the additive average of the homogeneity based affinity, $w_{i j}(3)$ and the object-feature based affinity $w_{i j}^{p}$, which yields

$$
W_{i j}=\omega_{1}\left(w_{i j}\right)+\omega_{2}\left(w_{i j}^{p}\right)
$$

where $\omega_{1}$ and $\omega_{2}$ are the trade-off values that satisfy $\omega_{1}+\omega_{2}=1$ of the normalized values of both $w$. 


\section{Experiments}

The proposed weighting function in random walks is tested on several medical images that mainly made up of the brain tumor images. In the experiments, the neighborhood radius $R$ is set at 3 with neighborhood size of 28 to avoid imposing excessive computational burden. The elements in generating the GLCM are consistently set across images, where the grey level quantization $G$, window size, displacement, and orientation were experimentally set to 128,3 by 3,1 , and averaging direction respectively. Although it was found to be unnecessary of having $G \geq 64$ in [19], but to safeguard the detail in medical images, higher $G$ is used. Small window size is chosen to ensure the accuracy in locating the boundaries between regions [20] and at the same time to keep the computational power low.

Performance of the proposed method is first demonstrated on the medical images that possess the faint boundaries or/ and textural elements. The ground truth images obtained from the field experts are projected as benchmarks in this experiment. Results of the proposed approach are compared with the original random walks algorithm. Minimal user interaction with as little seeds as possible is used to test the robustness of the methods. Figure 2 illustrates some of the experimental results. It can be seen that results using the proposed weighting function are considerably better as compared to the conventional random walks with the identical $R$. The results visually demonstrated the effectiveness of the proposed modifications of the weighting function. From Figure 2, it is observed that, the original random walks algorithm is less efficient when the gray levels of the regions do not appear to be homogenous. In contrast to that, with the proposed edge weight that penalizes the high differences within a local region and incorporating probability density using the GLCM variance, regions with random or orderliness changes of gray level are segmented relatively better.

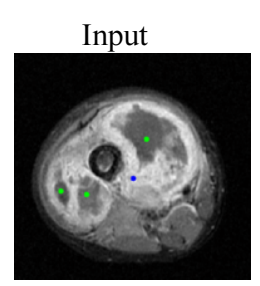

(a) Osteosarcoma

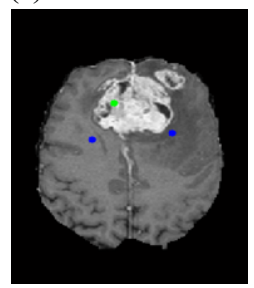

(b) Brain Tumor

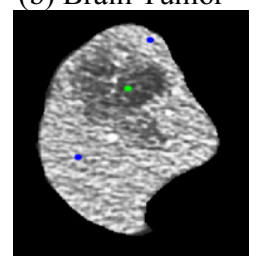

(c) Liver Tumor
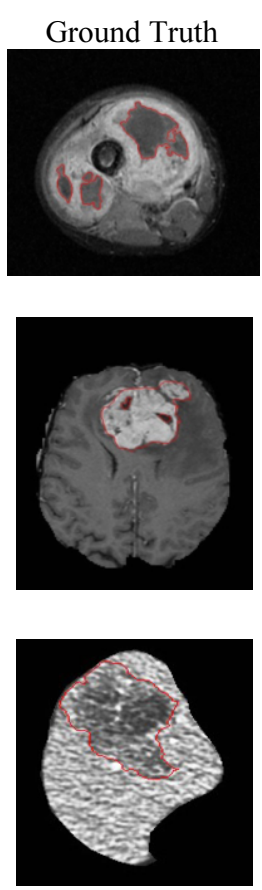
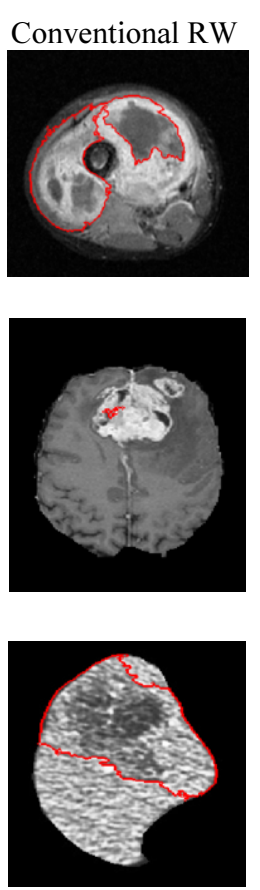
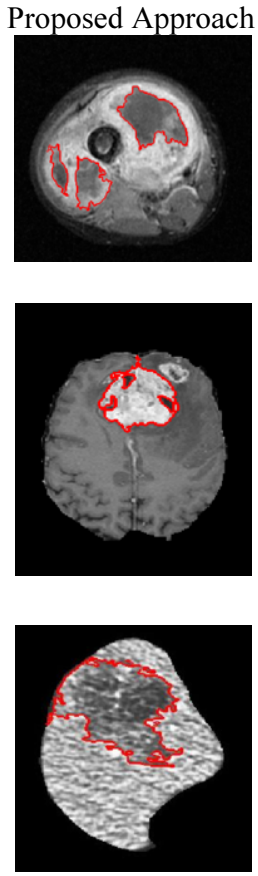

Fig. 2. Representative results using the conventional random walks and the proposed edge weight based on the limited user inputs (ROI in green and background in blue). 
Table 1

DICE, TPF, FPF, EPF using (a) conventional and (b) modified weighting function in random walks

\begin{tabular}{lllllllll}
\hline & DICE & \multicolumn{3}{l}{ TPF } & FPF & EFF \\
\cline { 2 - 8 } & (a) & (b) & (a) & (b) & (a) & (b) & (a) & (b) \\
\hline Dataset1 & 0.73044 & 0.78788 & 0.60027 & 0.65536 & 0.00005 & 0.00004 & 0.00152 & 0.00113 \\
Dataset2 & 0.90052 & 0.93482 & 0.90073 & 0.87564 & 0.00047 & 0.00060 & 0.02344 & 0.03410 \\
Dataset3 & 0.47090 & 0.62686 & 0.47900 & 0.55200 & 0.00166 & 0.00074 & 0.43300 & 0.18200 \\
Dataset4 & 0.72304 & 0.82048 & 0.62038 & 0.72152 & 0.00094 & 0.00127 & 0.01602 & 0.02146 \\
Dataset5 & 0.89372 & 0.93714 & 0.98354 & 0.95162 & 0.00536 & 0.00326 & 0.23319 & 0.13918 \\
Dataset6 & 0.80795 & 0.81320 & 0.89283 & 0.88016 & 0.00425 & 0.00372 & 0.32544 & 0.28384 \\
Mean & 0.75443 & 0.82006 & 0.74612 & 0.77272 & 0.00212 & 0.00161 & 0.17210 & 0.11029 \\
Std & 0.15847 & 0.11427 & 0.20505 & 0.15444 & 0.00218 & 0.00152 & 0.18486 & 0.11109 \\
\hline
\end{tabular}

To further validate the proposed method, images of brain tumor [21] are employed. The images, either the $\mathrm{T} 2$ or $\mathrm{T} 1 \mathrm{C}$ that are found to be independently sufficient to identify tumor are preselected. The segmented results are then compared with the ground truth. Table 1 shows the results that comprise the mean scores of DICE index, True Positive Fraction (TPF), False Positive Fraction (FPF), and Error Positive Fraction (EPF) of each brain dataset made up of 174 images.

The proposed model performs statistically better with higher DICE and TPF along with lower FPF and $\mathrm{EFF}$ in comparison to the conventional random walks.

\section{Conclusion}

In this paper, the weighting function that considers a more exhaustive relationship between the neighboring nodes is proposed. Extra terms that include standard deviation of the local pixels within the adjacent pixels and the object feature based affinity are incorporated in to the weighting function. The method is tested and the results have demonstrated a better ROI segmentation as compared to the traditional random walks by using several evaluation approaches. We are currently extending this work to quantitatively measure the performance of the segmentation with the images of brain tumor [21].

\section{Acknowledgement}

This work is supported by the grant of Spatially Aware Clustering Models for Multispectral Image Segmentation (1001/PKOMP/817067).

\section{References}

[1] L. Grady, Random walks for image segmentation, IEEE Transactions on Pattern Analysis and Machine Intelligence 28 (2006), 1768-1783.

[2] F. Maier, A. Wimmer, G. Soza, J. N. Kaftan, D. Fritz and R. Dillmann, Automatic liver segmentation using the random walker algorithm, in: Bildverarbeitung FÜR Die Medizin, T. Tolxdorff, J. Braun, T. Deserno, A. Horsch, H. Handels and H.-P. Meinzer, eds., Springer, Berlin Heidelberg, 2008, pp. 56-61.

[3] P.Y. Baudin, N. Azzabou, P.G. Carlier and N. Paragios, Automatic skeletal muscle segmentation through random walks and graph-based seed placement, 2012 9th IEEE International Symposium on Biomedical Imaging (ISBI), 2012, 10361039. 
[4] P. Wighton, M. Sadeghi, T.K. Lee and M.S. Atkins, A fully automatic random walker segmentation for skin lesions in a supervised setting, The Proceedings of the 12th International Conference on Medical Image Computing and ComputerAssisted Intervention: Part II, London, UK, 2009, 1108-1115.

[5] L. Grady, Multilabel random walker image segmentation using prior models, IEEE Computer Society Conference on Computer Vision and Pattern Recognition 1 (2005), 763-770.

[6] S. El-Hilo, Y.T. Weldeselassie and M.S. Atkins, Second order DTMR image segmentation using random walker, Proc. SPIE, Medical Imaging 2011: Image Processing 7962 (2011), 79624I. doi: 10.1117/12.878216

[7] W.X. Yang, J.F. Cai, J.M. Zheng and J.B. Luo, User-friendly interactive image segmentation through unified combinatorial user inputs, IEEE Transactions on Image Processing 19 (2010), 2470-2479.

[8] L. Grady, T. Schiwietz, S. Aharon and R. Westermann, Random walks for interactive organ segmentation in two and three dimensions: Implementation and validation, Medical Image Computing and Computer-Assisted InterventionMICCAI 2005, Springer Berlin Heidelberg 3750 (2005), 773-780.

[9] S.P. Dakua and J.S. Sahambi, LV contour extraction using difference of gaussian weighting function and random walk approach, 2009 Annual IEEE in India Conference (INDICON) (2009), 1-4.

[10] S.P. Dakua and J.S. Sahambi, Weighting function in random walk based left ventricle segmentation, 2011 18th IEEE International Conference on Image Processing, 2011, 2133-2136.

[11] R. Rzeszutek, T. El-Maraghi and D. Androutsos, Image segmentation using scale-space random walks, 2009 16th International Conference on Digital Signal Processing, 2009, 1-4.

[12] L. Liu and G. Wang, Recursive Formulae for Hermite Polynomial Approximations to Rational Bézier Curves, presented at the Proceedings of the Geometric Modeling and Processing, 2000, 190-199.

[13] H. Su, Z. Su, S. Zheng, H. Yang and S. Wei, Interactive cell segmentation based on phase contrast optics, Bio-Medical Materials and Engineering 24 (2014), 29-35.

[14] L. Grady and M.-P. Jolly, Weights and topology: A study of the effects of graph construction on 3D image segmentation, The Proceedings of the 11th International Conference on Medical Image Computing and ComputerAssisted Intervention-Part I, New York, USA, 2008.

[15] A. Ghosh and S. Boyd, Growing well-connected graphs, in IEEE Conference on Decision \& Control, San Diego, CA, USA, 2006, pp. 6605-6611.

[16] K.C. Ciesielski and J.K. Udupa, Affinity functions in fuzzy connectedness based image segmentation II: Defining and recognizing truly novel affinities, Computer Vision and Image Understanding 114 (2010), 155-166.

[17] G. Castellano, L. Bonilha, L.M. Li and F. Cendes, Texture analysis of medical images, Clinical Radiology 59 (2004), 1061-1069.

[18] S. Hu, C. Xu, W. Guan, Y. Tang and Y. Liu, Texture feature extraction based on wavelet transform and gray-level cooccurrence matrices applied to osteosarcoma diagnosis, Bio-Medical Materials and Engineering 24 (2014), 129-143.

[19] D.A. Clausi, An analysis of co-occurrence texture statistics as a function of grey level quantization, Canadian Journal of Remote Sensing 28 (2002), 45-62.

[20] M. De Martinao, F. Causa and S.B. Serpico, Classification of optical high resolution images in urban environment using spectral and textural information, Proceedings of 2003 IEEE International Geoscience and Remote Sensing Symposium 1 (2003), 467-469..

[21] Medical Image Computing and Computer Assisted Intervention (MICCAI), Multimodal Brain Tumor Segmentation, http://www2.imm.dtu.dk/projects/BRATS2012/data.html, 2012. 